\title{
THE ORIGIN OF THE BIFURCATION IN THE SAGITTARIUS STREAM
}

\author{
M. Fellhauer ${ }^{1}$ V. Belokurov, ${ }^{1}$ N. W. Evans, ${ }^{1}$ M. I. Wilkinson, ${ }^{1}$ D. B. Zucker, ${ }^{1}$ G. Gilmore, ${ }^{1}$ \\ M. J. Irwin, ${ }^{1}$ D. M. Bramich, ${ }^{1}$ S. Vidrih, ${ }^{1}$ R. F. G. Wyse, ${ }^{2}$ T. C. BeErs, ${ }^{3}$ and J. Brinkmann, ${ }^{4}$ \\ Received 2006 May 1; accepted 2006 June 20
}

\begin{abstract}
The latest Sloan Digital Sky Survey data reveal a prominent bifurcation in the distribution of debris of the Sagittarius dwarf spheroidal (Sgr) beginning at a right ascension of $\alpha \approx 190^{\circ}$. Two branches of the stream (A and B) persist at roughly the same heliocentric distance over at least $50^{\circ}$ of arc. There is also evidence for a more distant structure (C) well behind the A branch. This paper provides the first explanation for the bifurcation. It is caused by the projection of the young leading (A) and old trailing (B) tidal arms of the Sgr, while the old leading arm (C) lies well behind A. This explanation is only possible if the halo is close to spherical, as the angular difference between the branches is a measure of the precession of the orbital plane.
\end{abstract}

Subject headings: galaxies: dwarf — galaxies: individual (Sgr dSph) — Galaxy: halo — Galaxy: structure

\section{INTRODUCTION}

The disrupting Sagittarius dwarf spheroidal galaxy (Sgr) was discovered by Ibata et al. (1994). It was soon realized that the Sgr provided a powerful tool for the study of the Galaxy (Ibata et al. 1997). The nucleus of the Sgr has survived for many orbits around the Galaxy, while its tidal tails have now been detected over a full $360^{\circ}$ on the sky (see e.g., Totten \& Irwin 1998; Majewski et al. 2003; Belokurov et al. 2006). The disrupted fragments of the Sgr diffuse in the Galactic potential. As the pericenter of the Sgr's orbit is $\sim 16 \mathrm{kpc}$, while its apocenter is $\sim 60 \mathrm{kpc}$, the debris provides a strong constraint on the Galaxy's halo.

The morphology of the Sgr stream is known in detail in the Galactic southern hemisphere thanks to 2MASS (Majewski et al. 2003, 2004; Skrutskie et al. 2006). Ibata et al. (2001a) and Newberg et al. $(2002,2003)$ made early detections of Sgr tidal debris in data from the Sloan Digital Sky Survey (SDSS; see Hogg et al. 2001; Stoughton et al. 2002; Smith et al. 2002; Pier et al. 2003; Ivezić et al. 2004; Gunn et al. 2006). Then, Belokurov et al. (2006) used a color cut to pick out the upper main-sequence and turnoff stars in SDSS Data Release 5 (DR5; AdelmanMcCarthy et al. 2006) belonging to the stream. In the Galactic northern hemisphere, they found a prominent bifurcation, or branching, in the stream, beginning at a right ascension $\alpha \approx$ $190^{\circ}$ (see the top-right panel of Fig. 1). The lower and upper declination branches of the stream, labeled A and B, can be traced until right ascensions of at least $\alpha \approx 140^{\circ}$. Using the location of the subgiant branch as an estimator, the A and B branches are reckoned to be at similar distances. There is also evidence in the data of a fainter, still more distant stream (C) directly behind the A branch.

Belokurov et al.'s (2006) data set is important for two reasons. First, it traces the Sgr stream around the North Galactic Cap, the very spot at which oblate and prolate dark halos give different

\footnotetext{
1 Institute of Astronomy, University of Cambridge, Madingley Road, Cambridge CB3 0HA, UK; madf@ast.cam.ac.uk, vasily@ast.cam.ac.uk,nwe@ast cam.ac.uk.

${ }^{2}$ The Johns Hopkins University, 3701 San Martin Drive, Baltimore, MD 21218

3 Department of Physics and Astronomy, CSCE: Center for the Study of Cosmic Evolution, and JINA: Joint Institute for Nuclear Astrophysics, Michigan State University, East Lansing, MI 48824.

4 Apache Point Observatory, P.O. Box 59, Sunspot, NM 88349.
}

predictions (e.g., Helmi 2004a). Second, the debris in 2MASS is dynamically younger than that found in SDSS, so the SDSS data should give stronger constraints, as the stars have had longer to move in the Galactic potential.

Early explorations of the evolution of the Sgr suggested that the Galactic halo may be close to spherical (Ibata et al. 2001b; Majewski et al. 2003). For example, Johnston et al. (2005) showed that the precession apparent in Sgr debris in the 2MASS data set strongly favored mildly oblate halos. However, Helmi (2004a) pointed out that many of the earlier data sets are restricted to stars that have only recently been torn off the Sgr and so have not diffused in the Galactic potential. In fact, Helmi (2004b) argued that the velocity measurements of 2MASS M giants in the leading arm favor strongly prolate halos, and this was subsequently confirmed by Law et al. (2005). Hence, present studies of the disruption of the Sgr have reached an impasse, with different data sets pointing to dramatically different flattenings.

Here, our aim is to show how the newly discovered bifurcation arises. Using numerical simulations, we argue that the complex morphology of the Sgr stream uncovered by Belokurov et al. (2006) can only be reproduced if the Galaxy's halo is close to spherical. Although our models do not resolve the contradiction between the precession rate and the velocities in the leading arm, they do provide a new and powerful argument in favor of an almost spherical Galaxy halo.

\section{THE SIGNIFICANCE OF THE BIFURCATION}

The results of a typical simulation of the tidal disruption of the Sgr in a nearly spherical potential are shown in Figure 1. We will give the details of the simulation setup shortly, but at the moment our aim is to gain a qualitative understanding of why the bifurcation occurs. The particles in Figure 1 are color coded according to when they were torn off the Sgr. In the direction of the SDSS DR5 data (namely, the opening angle defined by the green lines) there are four distinct streams of material. They are the young leading arm (labeled A), the old trailing arm (B), the old leading arm (C) and the young trailing arm (D). Here, old and young indicate when the stars were torn off. Three out of the four streams are identified in the SDSS DR5 data set. Stars belonging to the D stream are more difficult to detect, as they occur in DR5 primarily in the range $180^{\circ} \lesssim \alpha \lesssim 220^{\circ}$, where they are not easy to untangle from the other streams. The simulation data are 


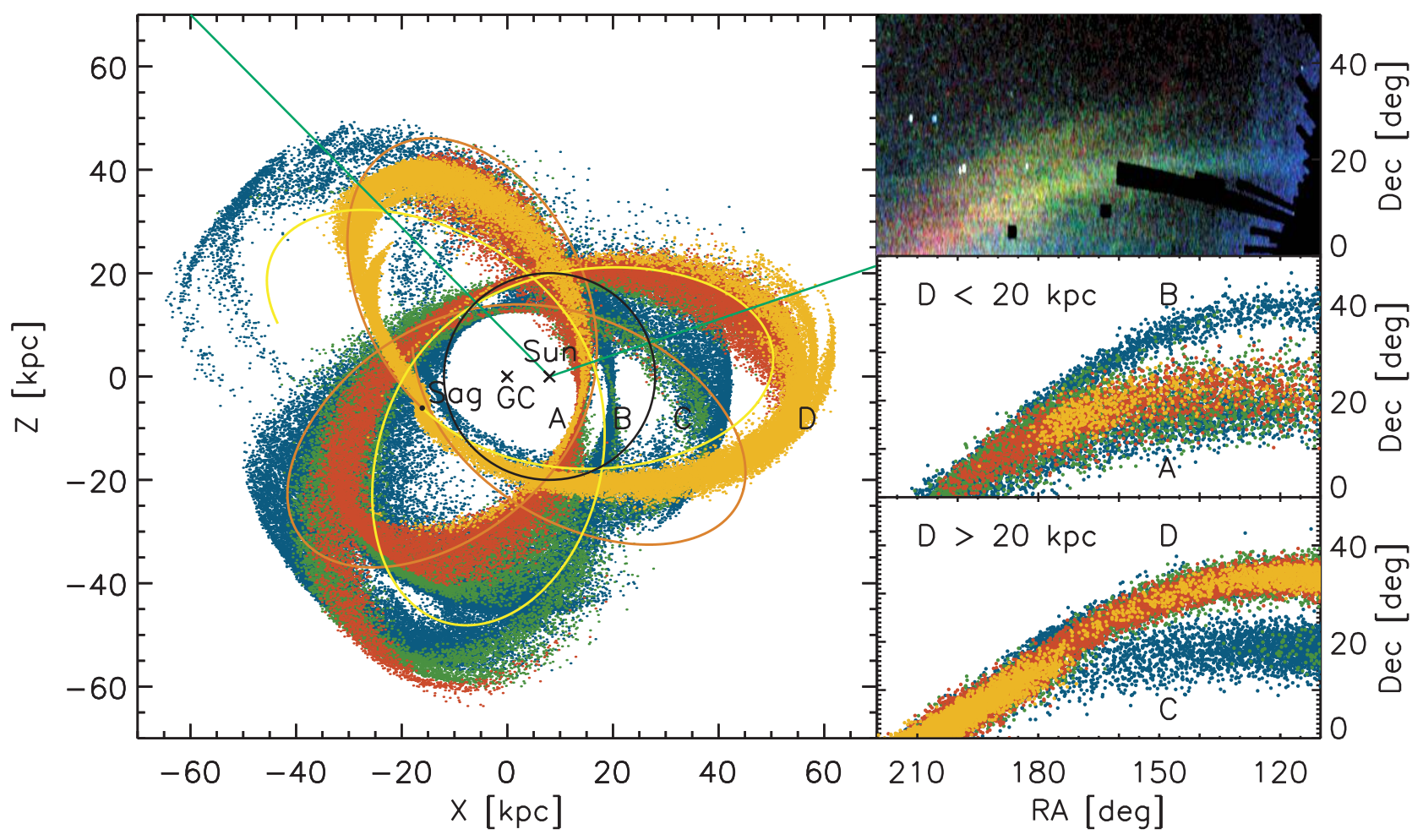

Fig. 1. - Left: Simulation showing the tails of the Sgr dSph. Particles are color coded according to when they were lost (gold: $<4$ Gyr ago, red: between 4 and $5.7 \mathrm{Gyr}$, green: between 5.7 and $7.4 \mathrm{Gyr}$, and blue: $>7.4 \mathrm{Gyr}$ ago). The yellow (orange) curves show the past (future) behavior of the Sgr's orbit over $2 \mathrm{Gyr}$. The orbital period is $0.7 \mathrm{Gyr}$. The positions of the Galactic center (GC), the Sun, and Sagittarius (Sgr) are marked. The green lines show the right ascension range $110^{\circ}<\alpha<220^{\circ}$, which corresponds to the SDSS data analyzed by Belokurov et al. (2006). The four streams are marked A (young leading arm), B (old trailing arm), C (old leading arm), and D (young trailing arm). The circle gives the distance cutoff at $20 \mathrm{kpc}$. Top right: The SDSS data from Belokurov et al. (2006), with stars color coded according to magnitude. Middle and bottom right: Scatter plots in right ascension and declination of the tidal debris. Only particles within (beyond) a heliocentric distance of $20 \mathrm{kpc}$ are plotted in the middle (bottom) panel. The black squares show the field locations of Belokurov et al. (2006). In the middle panel, streams A and B are clearly visible. The upper arm is the old trailing material, while the lower arm is the young leading material. In the bottom panel, the old leading material is in the lower and the young trailing material in the upper branch. ( The simulation uses a Miyamoto-Nagai disk and logarithmic halo with $q_{\phi}=1.05$, together with the set $\mathrm{d}$ of proper motions. The mass of $\mathrm{Sgr}$ is $10^{8} M_{\odot}$.)

separated according to heliocentric distance and then projected onto the sky as viewed from the Sun, as shown in the lower right panels of Figure 1. The young leading arm provides branch $\mathrm{A}$ and the old trailing arm branch B of the bifurcated stream of Belokurov et al. (2006). These two narrow branches are at similar heliocentric distances, as required to match the data. The material in these branches is about two revolutions apart in orbital phase. For the material beyond $20 \mathrm{kpc}$, the older leading material is in the lower declination branch, while the younger trailing material is in the upper. The old leading arm $(\mathrm{C})$ provides the more distant and fainter stream detected by Belokurov et al. (2006) behind the A branch. The young trailing arm (D) lies behind the B branch and so is not the structure seen by Belokurov et al. (2006).

The Sun lies roughly in the orbital plane of the Sgr. If the potential were exactly spherical, the debris of the Sgr would lie in a single plane and no bifurcation would exist. Any asphericity (whether intrinsic to the halo or produced by the bulge and disk) causes the orbital plane to precess and therefore the planes of the four arms to be slightly different. The positional difference between branches $\mathrm{A}$ and $\mathrm{B}$ is a direct measure of the precession over two orbital revolutions and hence the asphericity of the potential. The facts that (1) branches A and B are so close in projection and (2) branch $C$ lies behind branch A suggest that the precession is small, and that the potential is close to spherical. If the halo is too oblate or prolate, then debris is scattered over a wide range of locations and does not lie in thin, almost over- lapping streams on the sky. To back up this qualitative argument, let us now describe a suite of simulations developed to measure the properties of the bifurcation as a function of halo flattening, Sgr mass and proper motion.

\section{SIMULATIONS}

\subsection{Setup}

The present position of the Sgr dSph is $(\alpha, \delta)=\left(283^{\circ} .7,-30^{\circ} .5\right)$, while its heliocentric distance is $25 \pm 2 \mathrm{kpc}$ and radial velocity is $137 \mathrm{~km} \mathrm{~s}^{-1}$ (Ibata et al. 1997). Listed in Table 1 are two measurements of the proper motion of the Sgr, the first from Irwin et al. (1996) using Schmidt plates and the second from Ibata et al. (2001b) using Hubble Space Telescope (HST) data. Dinescu et al.'s (2005) recent measurement agrees with that of Irwin et al. (1996) within the errors. Given a choice of proper motions, we integrate back in time for $10 \mathrm{Gyr}$, adopting a potential for the Galaxy. At the final position, we insert a Plummer sphere containing $10^{6}$ particles with a scale length of $350 \mathrm{pc}$. We investigate models with a total mass of between $10^{8}$ and $10^{9} M_{\odot}$. The particles are integrated forward using the particle-mesh $N$-body code SUPERBOX (Fellhauer et al. 2000) until the position today is reached again.

While the present position of the Sgr is unchanged in all our simulations, the range of proper motions recorded in Table 1 is investigated. For the Galactic potential, we use one of two 
TABLE 1

Sets of Proper Motions Used in the Simulations

\begin{tabular}{|c|c|c|c|c|}
\hline Label & $\begin{array}{c}\mu_{\alpha} \cos \delta \\
\left(\mathrm{mas} \mathrm{yr}^{-1}\right)\end{array}$ & $\begin{array}{c}\mu_{\delta} \\
\left({\left.\operatorname{mas~} \mathrm{yr}^{-1}\right)}\right)\end{array}$ & $\begin{array}{c}v_{\mathrm{rad}} \\
\left(\mathrm{km} \mathrm{s}^{-1}\right)\end{array}$ & Remarks \\
\hline $\mathrm{a} \ldots \ldots \ldots \ldots \ldots \ldots \ldots \ldots \ldots \ldots$ & -2.65 & -0.88 & 137 & $H S T$ measurement ( Ibata et al. 2001b) \\
\hline $\mathrm{b}$ & -2.8 & -1.4 & 137 & Schmidt plates measurement (Irwin et al. 1996) \\
\hline $\mathrm{c}$ & -2.9 & -1.5 & 137 & $<1 \sigma$ variation of Schmidt plates \\
\hline 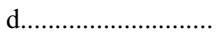 & -3.02 & -1.49 & 137 & Simulation fit from Law et al. (2005) \\
\hline $\mathrm{e}$ & -3.05 & 1.28 & 137 & $5 \sigma$ variation of $H S T$ values \\
\hline
\end{tabular}

possibilities. In the first (denoted by ML), the halo is represented by a logarithmic potential of the form

$$
\Phi_{\text {halo }}(r)=\frac{v_{0}^{2}}{2} \ln \left(R^{2}+z^{2} q_{\phi}^{-2}+d^{2}\right),
$$

with $v_{0}=186 \mathrm{~km} \mathrm{~s}^{-1}$ and $d=12 \mathrm{kpc}$ (where $R$ and $z$ are cylindrical coordinates). The parameter $q_{\phi}$ is the axis ratio of the equipotentials. It controls whether the halo is spherical $\left(q_{\phi}=1\right)$, oblate $\left(q_{\phi}<1\right)$, or prolate $\left(q_{\phi}>1\right)$. In general, $q_{\phi}$ is of course not the same as the axis ratio in the density $q$, which varies with radius for the logarithmic potential (see e.g., Evans 1993). The disk is represented by a Miyamoto-Nagai potential,

$$
\Phi_{\text {disk }}(R, z)=\frac{G M_{d}}{\sqrt{R^{2}+\left(b+\sqrt{z^{2}+c^{2}}\right)^{2}}},
$$

with $M_{d}=10^{11} M_{\odot}, b=6.5 \mathrm{kpc}$, and $c=0.26 \mathrm{kpc}$. Finally, the bulge is modeled as a Hernquist potential,

$$
\Phi_{\text {bulge }}(r)=\frac{G M_{b}}{r+a},
$$

using $M_{b}=3.4 \times 10^{10} M_{\odot}$ and $a=0.7 \mathrm{kpc}$. The superposition of these components gives quite a good representation of the Milky Way. The circular speed at the solar radius is $\sim 220 \mathrm{~km} \mathrm{~s}^{-1}$. The major advantage is the analytical accessibility of all quantities (forces, densities, and so on). Hence, this model has been very widely used, particularly in many previous investigations of the Sgr stream (e.g., Helmi 2004a, 2004b; Johnston et al. 2005; Law et al. 2005).

In the second (denoted by DB), we use the Galactic potential suggested by Dehnen \& Binney (1998). It consists of three disk

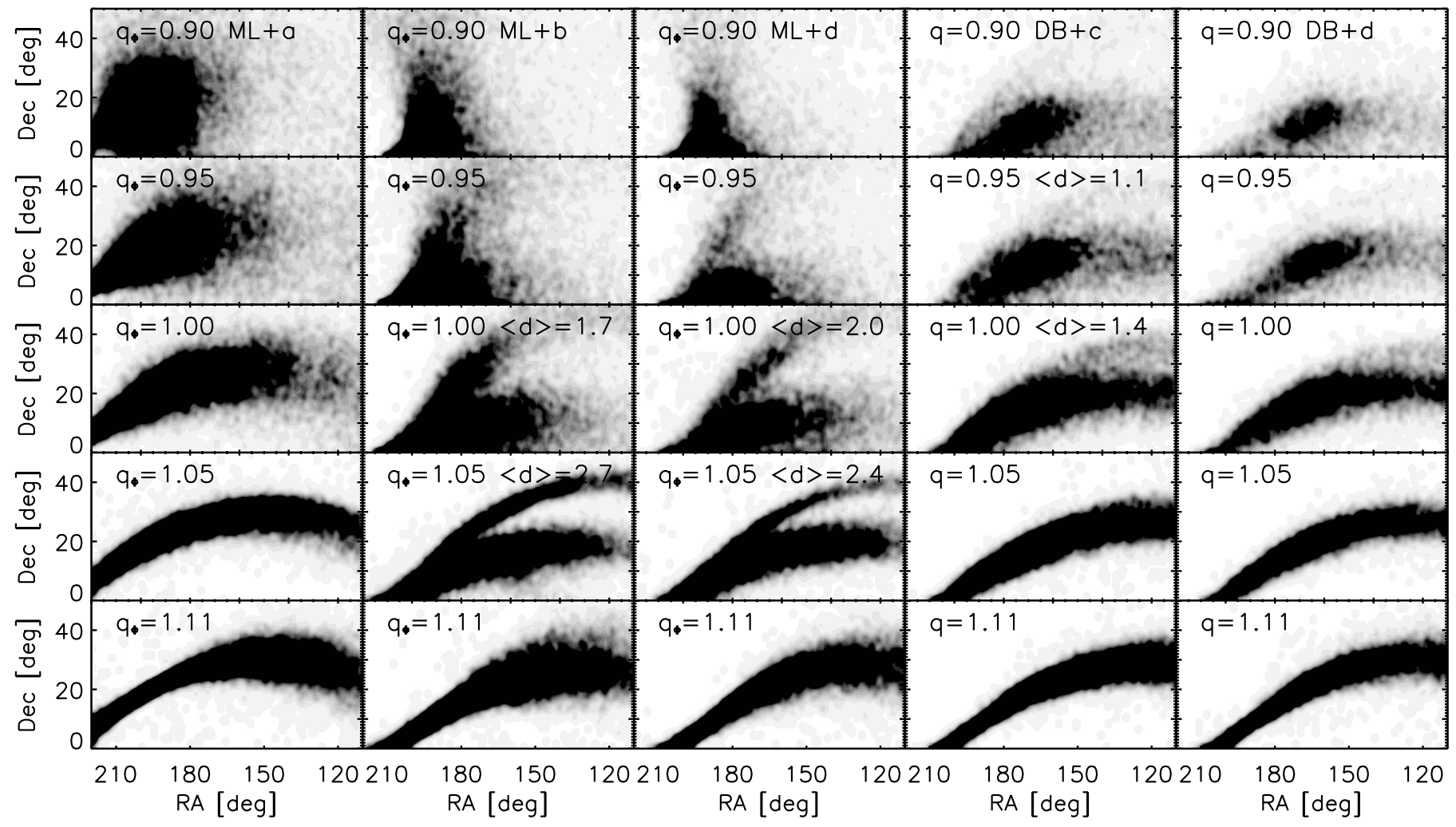

FIg. 2.- Projected density of the young leading and old trailing tidal debris of the Sgr in a sequence of simulations with different halo flattenings, proper motions, and Galaxy models. The key at the top of each column has the following significance: ML means Miyamoto-Nagai disk and logarithmic halo models, DB means the Dehnen \& Binney models, while a, b, c, d, or e refers to the choice of proper motions. The panels in a given column differ only in the flattening of the halo, with $q_{\phi}$ for the ML models and $q$ for the DB models recorded in the upper left corner. The mass of the Sgr is $10^{8} M_{\odot}$. Note that the particles in the simulation represent both stars and dark matter. 
TABLE 2

The Strength and Range of the Bifurcation in Nearly Spherical Haloes

\begin{tabular}{|c|c|c|c|c|c|c|c|c|c|c|}
\hline \multirow[b]{3}{*}{ Model } & \multirow[b]{3}{*}{$Q_{\phi}$} & \multicolumn{9}{|c|}{ Proper Motions } \\
\hline & & \multirow{2}{*}{$\stackrel{\mathrm{a}}{-}$} & \multicolumn{2}{|c|}{$\mathrm{b}$} & \multicolumn{2}{|c|}{$\mathrm{c}$} & \multicolumn{2}{|c|}{$\mathrm{d}$} & \multicolumn{2}{|c|}{ e } \\
\hline & & & $\langle d\rangle$ & $\alpha_{0}$ & $\langle d\rangle$ & $\alpha_{0}$ & $\langle d\rangle$ & $\alpha_{0}$ & $\langle d\rangle$ & $\alpha_{0}$ \\
\hline 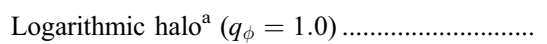 & 0.92 & $\ldots$ & 1.7 & 170 & 1.9 & 180 & 2.0 & 190 & 1.5 & 160 \\
\hline 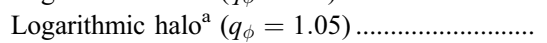 & 0.95 & $\ldots$ & 2.7 & 190 & 2.3 & 190 & 2.4 & 170 & 1.7 & 150 \\
\hline Dehnen \& Binney models ${ }^{\mathrm{b}}(q=0.95) \ldots \ldots \ldots \ldots . . .$. & 0.95 & $\ldots$ & 1.1 & 160 & 1.1 & 150 & $\ldots$ & $\ldots$ & $\ldots$ & $\ldots$ \\
\hline 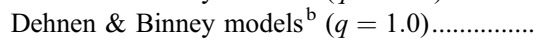 & 0.97 & $\ldots$ & 1.4 & 130 & 1.4 & 150 & $\ldots$ & $\ldots$ & $\ldots$ & $\ldots$ \\
\hline
\end{tabular}

a All Galactic models with $q_{\phi}=0.8,0.9,0.95,1.0,1.05,1.11,1.25$, and 1.5 were investigated. If there is no bifurcation, or if the lower branch of the bifurcation bends back to negative declinations, the model is discarded. For models in which there is a bifurcation, the strength $\langle d\rangle$ and the onset $\alpha_{0}(\mathrm{deg})$ are given for each set of proper motions.

b All Galactic models with $q=0.8,0.9,0.95,1.0,1.05,1.11,1.25$, and 1.5 were investigated, but only those with bifurcations are reported.

components (namely, the ISM, the thin, and the thick disk), each of the form

$$
\rho_{\text {disk }}(R, z)=\frac{\Sigma_{d}}{2 z_{d}} \exp \left(-\frac{R_{m}}{R}-\frac{R}{R_{d}}-\frac{|z|}{z_{d}}\right)
$$

With $R_{m}=0$, equation (4) describes a standard double exponential disk with scale length $R_{d}$, scale height $z_{d}$, and central surface density $\Sigma_{d}$. For the stellar disks, $R_{m}$ is set to zero, while for the ISM disk, we allow for a central depression by setting $R_{m}=$ $4 \mathrm{kpc}$. Furthermore, the halo and the bulge are represented by two spherical density distributions of the form

$$
\rho_{\mathrm{S}}(R, z)=\rho_{0}\left(\frac{m}{r_{0}}\right)^{-\gamma}\left(1+\frac{m}{r_{0}}\right)^{\gamma-\beta} \exp \left(-\frac{m^{2}}{r_{t}^{2}}\right)
$$

where $m^{2}=R^{2}+z^{2} q^{-2}$ and $q$ is the axis ratio in the density. We choose the parameters according to the best-fit model 4 in Dehnen \& Binney (1998). This provides a better representation of the Galaxy, but at somewhat greater computational cost.

\subsection{Results}

Snapshots of the distribution of tidal debris around the north Galactic cap for some typical simulations are shown in Figure 2. We quickly see that most of the models do not look at all like the data. Only halos close to spherical provide bifurcated streams. The simulated streams in moderately and strongly oblate or prolate halos do not bifurcate.

To proceed further, we need to develop an objective criterion for identifying the bifurcation. We use a Marquand-Levenberg routine to fit a single Gaussian and two Gaussians to the declination distribution in the young leading and old trailing tidal debris. Models for which a single Gaussian is everywhere preferred (as judged by the $\Delta \chi^{2}$ ) are unacceptable, as they do not show two identifiable streams. If two Gaussians are a better fit than a single, then the ratio $d$ of the distance between the two peaks to the sum of the dispersions of each peak is computed as a function of right ascension. We refer to the mean value of this parameter, taken over all right ascensions $110^{\circ} \leq \alpha \leq 220^{\circ}$, as the strength of the bifurcation, $\langle d\rangle$. The onset of the bifurcation $\alpha_{0}$ is taken to be the right ascension when $d=1.5$. Applying this algorithm to Belokurov et al.'s (2006) data set, the bifurcation has strength $\langle d\rangle \approx 1.7$ and begins at a right ascension $\alpha_{0} \approx 190^{\circ}$. For a range of simulations, the same quantities are recorded in Table 2. Both Galaxy models contain a flattened disk and bulge, so the model parameters $\left(q_{\phi}\right.$ and $\left.q\right)$ are not a reliable guide to the overall flat- tening of the potential. Rather, we give in Table 2 the axis ratio of the equipotentials $Q_{\phi}$ at the mean of the pericentric and apocentric distances of the Sgr's orbit.

There are a number of interesting conclusions from the Table. First, very few models actually give bifurcations at all. Of the 80 models tested, only 10 give bifurcated streams. A bifurcation occurs if the axis ratio of the potential $Q_{\phi}$ lies in the range $0.92 \lesssim Q_{\phi} \lesssim 0.97$. The best overall match to the data is given
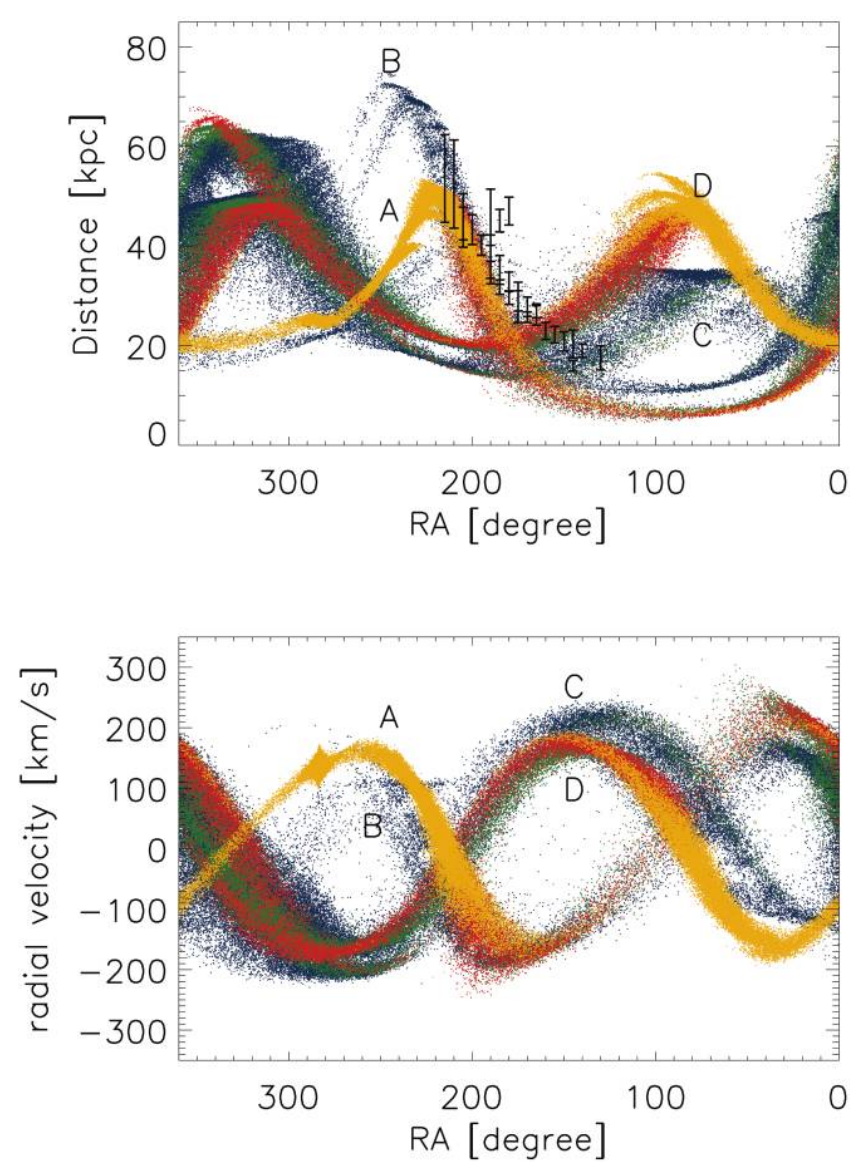

FIG. 3.-Plots of the heliocentric distance and velocity vs. right ascension for the same model as in Fig. 1. Again, particles are color coded according to when they were lost (gold: <4 Gyr ago, red: between 4 and 5.7 Gyr, green: between 5.7 and $7.4 \mathrm{Gyr}$, and blue: $>7.4 \mathrm{Gyr}$ ago). The data points give the heliocentric distances to the streams, as derived from fitting the subgiant branch described in Belokurov et al. (2006). Note that the distances to the A and B streams are too small for right ascensions $\alpha \lesssim 190^{\circ}$. 


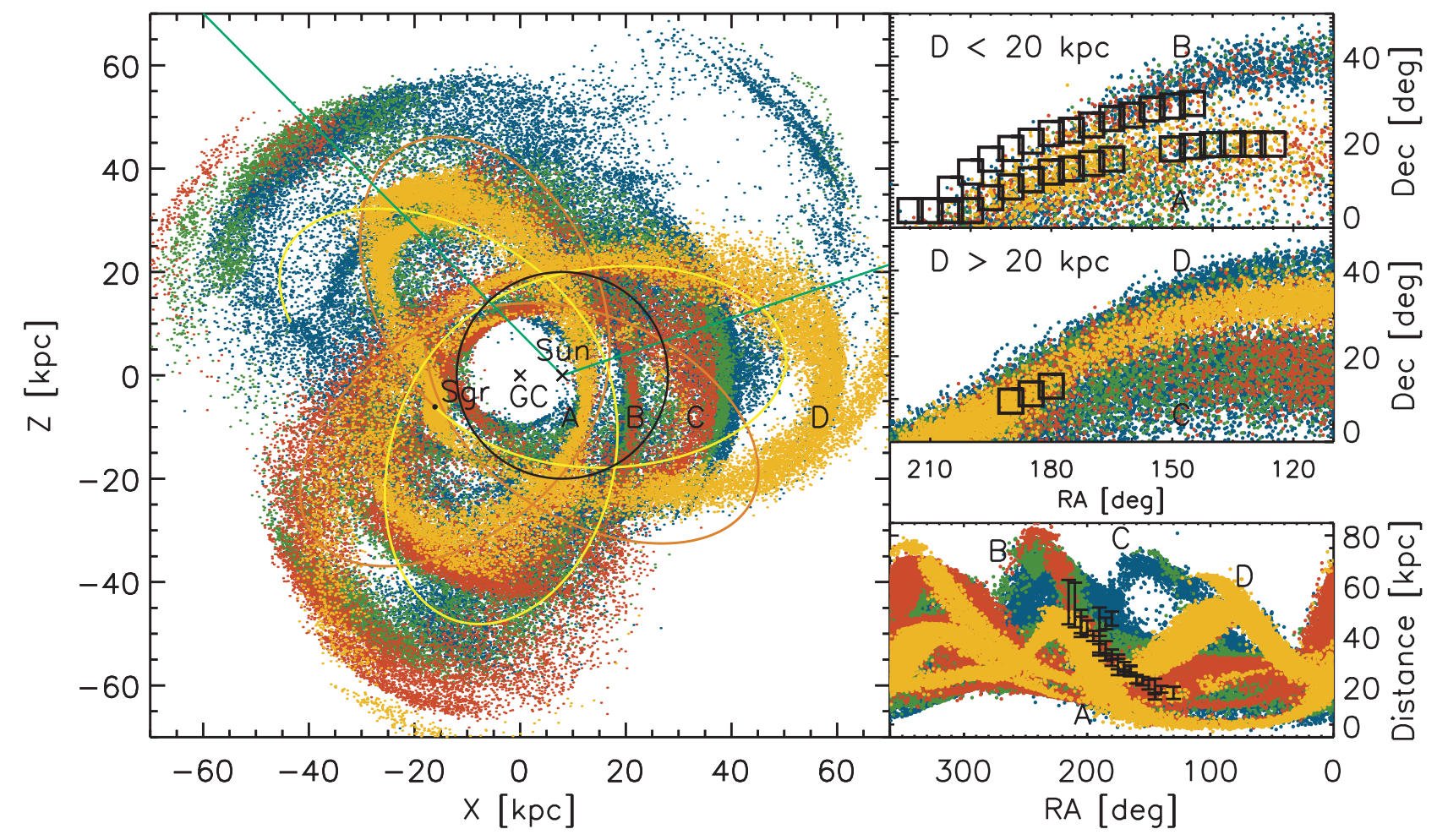

FIG. 4.-As in Fig. 1, but now the mass of the Sgr has been increased to $5 \times 10^{8} M_{\odot}$. A bifurcation is visible in the right-hand panels, but it is less dramatic than in the data.

by simulations using the Miyamoto-Nagai disk and logarithmic halo with $q_{\phi}=1$, together with sets c or $\mathrm{d}$ of proper motions. They reproduce the strength and the location of the bifurcation reasonably well. Second, if we use the proper motions measured by HST (set a, derived by Ibata et al. 2001b), we do not obtain bifurcated streams, whatever the halo flattening. The precession is controlled not just by the flattening of the potential, but also by the eccentricity of the orbit, and hence the proper motions. Sets $b$ and $\mathrm{c}$ of proper motions, with values close to those derived from Schmidt plates (Irwin et al. 1996), provide much better fits. Third, Helmi (2004b) has claimed that strongly prolate halos with $q \approx 1.65$ give the best fit to the 2MASS data on the right ascension, declination, heliocentric distance, and radial velocity of the tidal debris. Such strongly prolate models do not match the bifurcated stream in the SDSS data set. In fact, the claims of prolateness rely heavily on their choice of Galactic potential (Miyamoto-Nagai disk and logarithmic halo) and are not reproduced with the Dehnen \& Binney models. The MiyamotoNagai disk declines like a power law, rather than an exponential, and so it is reasonable to interpret the finding of prolateness or stretching of the halo as compensation for deficiencies in the disk model. Similarly, although some of the nominally prolate halo models $\left(q_{\phi}>1\right)$ in Table 2 provide matches to the bifurcation data, the equipotentials are mildly oblate $\left(Q_{\phi}<1\right)$ at the radii probed by Sgr's orbit.

Nearly spherical models can reproduce the projected density of the Sgr stream around the northern Galactic cap. However, as is traditional in this area, our simulations do not fit all the data! For example, there is a mismatch of $\sim 20^{\circ}$ in the right ascension of the beginning of the $\mathrm{C}$ stream (cf. the upper and lower right panels of Fig. 1). Figure 3 shows the heliocentric distances and velocities of stream A and B for the simulation of Figure 1, together with the heliocentric distances derived by Belokurov et al. (2006) from subgiant branch fitting. The heliocentric dis- tances of the simulated streams are within the observational error bars over the range of right ascensions $\alpha \gtrsim 190^{\circ}$, but they are too small for $\alpha \lesssim 190^{\circ}$. In common with other simulations in oblate halos (see e.g., Johnston et al. 2005), the radial velocities of 2MASS M giants in the leading arm are also not matched. Possible causes of these discrepancies are discussed shortly.

The existence of the bifurcation also constrains the mass of the Sgr. For example, Figure 4 shows a simulation identical to that of Figure 1, but with the initial mass of the Sgr increased to $5 \times$ $10^{8} M_{\odot}$. The more massive the Sgr, the greater its internal velocity dispersion. This has two consequences: the tidal arms are broader, and they diffuse away from the Sgr's orbital path more quickly. The overall effect is that debris is scattered over a wider range of locations. As the subpanels on the right hand side show, the bifurcation persists but is less dramatic than in the data, while the $\mathrm{A}$ and $\mathrm{B}$ streams are no longer as collimated as in the data. The run of heliocentric distances with right ascensions, however, is a somewhat better match. Figure 5 shows a sequence of simulations with Sgr masses between $10^{8}$ and $10^{9} M_{\odot}$, while the Galactic potential is kept fixed as a Miyamoto-Nagai disk and logarithmic halo with $q_{\phi}=1.05$. The bifurcation blurs with increasing mass. Quantitatively, the strength of the bifurcation $\langle d\rangle$ falls from 2.4 when the Sgr's mass is $10^{8} M_{\odot}$ to 1.9 at $2.5 \times$ $10^{8} M_{\odot}$ and to 1.2 at $5 \times 10^{8} M_{\odot}$. Once the Sgr mass rises much above $5 \times 10^{8} M_{\odot}$, there is no visible bifurcation. High-mass models are disfavored until it has been demonstrated that the tidal streams of stars can remain as highly collimated as in the data. In Table 3, we give the properties of the Sgr remnant at the end of our simulations. Of course, our simulations show both dark matter particles and stars, whereas the observable today is the luminous matter left in the Sgr dSph. The mass loss of the Sgr is mainly affected by its orbit and hence the choice of proper motion together with the choice of Galactic potential. Since these are uncertain, we also give in Table 3 the internal crossing times 
TABLE 3

Properties of the Models of the SGr dSph

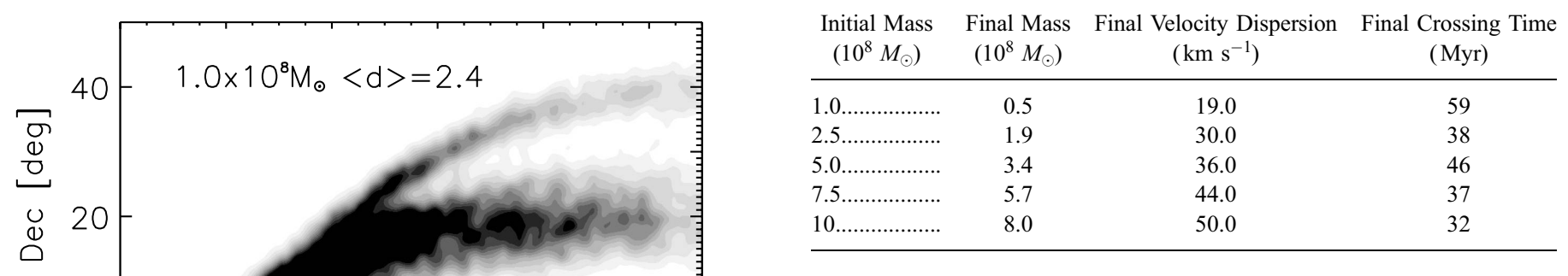

at the virial radius and the three-dimensional velocity dispersion, which governs the broadening of the tails.

Our overall picture gives three predictions. First, a second wrap of branch D may be detectable in the 2MASS data, closer than that already reported by Majewski et al. (2003). Second, the dynamically older B stream should have a larger velocity dispersion than the younger A stream. The mean velocities of branches $\mathrm{A}$ and B probably differ by $\sim 15 \mathrm{~km} \mathrm{~s}^{-1}$, although this may be hard to measure, as it may be less than the stream's internal dispersions. Third, if our current ideas on the star formation history of the Sgr are correct (e.g., Grebel 2000), then there may be a difference in the stellar populations of the streams. Stream A may contain evidence for a younger population that is not present in stream B.

\section{CONCLUSIONS}

The Sgr stream, as seen by the Sloan Digital Sky Survey (Belokurov et al. 2006), is composed of two branches (A and B) at about the same heliocentric distance, visibly diverging at right ascensions $\alpha \approx 190^{\circ}$ to give a bifurcation, together with a third stream (C) aligned with the A branch, but well behind it. This complex and intricate morphology throws down an enormous challenge to modelers.

Here, we have given a physical picture of how this structure may arise. The bifurcation is caused by a projection of the young leading (A) and the old trailing (B) tidal arms of the Sgr, while the old leading arm (C) lies well behind $\mathrm{A}$. The bifurcation between $\mathrm{A}$ and $\mathrm{B}$, and the positioning of $\mathrm{C}$ behind $\mathrm{A}$, can only be reproduced in simulations of the disruption of the Sgr if the halo is nearly spherical. Simulations in either moderately or strongly oblate or prolate halos fail these tests by a wide margin. In particular, the bifurcation only exists if the axis ratio of the potential $Q_{\phi}$ at the radii sampled by the Sgr's orbit lies in the range $0.92 \lesssim Q_{\phi} \lesssim 0.97$. The physical explanation of this is easy to provide. The material in the A and B branches is about two revolutions apart in orbital phase. The angular difference on the sky between the $\mathrm{A}$ and $\mathrm{B}$ branches is therefore a direct measure of the precession of the orbital plane of the Sgr over two revolutions. As the angular difference is small, so the precession of the orbital plane is small, and so the potential must be close to spherical. If the potential is moderately prolate or oblate, debris is scattered over a much wider range of locations. The path from observation to theoretical conclusion is surprisingly direct and independent of detailed modeling.

The bifurcation also provides a strong constraint on the mass

FIG. 5.- Sequence of simulations differing only in the mass of the Sgr dSph at the beginning of our $10 \mathrm{Gyr}$ simulations. This is marked in the top left-hand corner and varies from $2.5 \times 10^{8} M_{\odot}$ (top panel) to $10^{9} M_{\odot}$ (bottom panel $)$. If there is a bifurcation, then its strength $\langle d\rangle$ is also recorded. The Galactic model is a Miyamoto-Nagai disk and logarithmic halo with $q_{\phi}=1.05$, while proper motion set $\mathrm{d}$ is used. of the Sgr and its debris. If this is much larger than $5 \times 10^{8} M_{\odot}$, then the tidal streams are too diffuse to give a clear bifurcation. This also is easy to understand. As the internal velocity dispersion increases with progenitor mass, so the streams become broader and diffuse more quickly in the Galactic potential. The A and B branches do not then have the highly collimated appearance seen in the data. 
Our simulations, like all other simulations of the disruption of the Sgr in the literature, do not agree with all the data. In particular, the detailed distances to the A, B, and C streams given in Belokurov et al. (2006) are not reproduced over the full range of right ascension. Although this is a defect, the limitations of the commonly used methodology for Sgr disruption simulations also need to be acknowledged. The underlying assumption is that the Galactic potential is static and unevolving over up to $10 \mathrm{Gyr}$ timescales. This is clearly incorrect; the Milky Way is believed to have accreted $30 \%$ of its mass over the last 5 Gyr (see e.g., van den Bosch 2002; Neistein et al. 2006). Simulations typically show that the last major merger take place at about a redshift $z=1$, roughly 8 Gyr ago (Navarro et al. 1995). Although most of the mass will have accreted in the outer parts of the Galaxy, the Sgr's orbit extends out to $\sim 60 \mathrm{kpc}$ and will surely been affected by this rearrangement. The formation of the Galactic bar has been dated to between 5 and 8 Gyr ago (see e.g., Sevenster 1999a, 1999b), and so bar-driven evolution of the inner Galaxy will also have caused substantial changes. The effects of time evolution are of much greater importance for the SDSS data set than for the 2MASS data set, which is restricted to dynamically younger material.

The strength of the argument presented in this paper is that it relies on the gross morphological features of the Sgr stream. To reproduce the detailed positions and velocities of stars in the $\mathrm{A}, \mathrm{B}$, and $\mathrm{C}$ branches may well require a clearer understand of Galactic evolution. However, the existence of a bifurcation in nearly spherical potentials is a robust result. To challenge the main conclusion of this paper requires the devising of an alternative explanation of the existence of two streams that are closely matched in distance over a $\sim 50^{\circ}$ arc. In this respect, our argument compares favorably with other methods of determination of the halo shape using methods such as the flaring of the neutral gas layer (e.g., Olling \& Merrifield 2000) or the stellar kinematics of halo stars (e.g., van der Marel 1991). These are afflicted by sys- tematic uncertainties regarding the contribution of the cosmic ray pressure or the orientation of the stellar velocity ellipsoid, for example. In contrast, the bifurcation in the Sgr stream is a clean, simple, and direct test.

M. F., V. B., M. I. W., and D. Z. thank the Particle Physics and Astronomy Research Council of the UK for financial support, and Walter Dehnen for providing software. We thank Eric Bell, Heidi-Jo Newberg, Connie Rockosi, Brain Yanny, and Kathryn Johnston (the referee) for constructive comments on the draft version. Funding for the SDSS and SDSS-II has been provided by the Alfred P. Sloan Foundation, the Participating Institutions, the National Science Foundation, the US Department of Energy, the National Aeronautics and Space Administration, the Japanese Monbukagakusho, the Max Planck Society, and the Higher Education Funding Council for England. The SDSS Web Site is http://www.sdss.org/.

The SDSS is managed by the Astrophysical Research Consortium for the Participating Institutions. The Participating Institutions are the American Museum of Natural History, Astrophysical Institute Potsdam, University of Basel, Cambridge University, Case Western Reserve University, University of Chicago, Drexel University, Fermilab, the Institute for Advanced Study, the Japan Participation Group, The Johns Hopkins University, the Joint Institute for Nuclear Astrophysics, the Kavli Institute for Particle Astrophysics and Cosmology, the Korean Scientist Group, the Chinese Academy of Sciences (LAMOST), Los Alamos National Laboratory, the Max-PlanckInstitute for Astronomy (MPIA), the Max-Planck-Institute for Astrophysics (MPA), New Mexico State University, The Ohio State University, University of Pittsburgh, University of Portsmouth, Princeton University, the United States Naval Observatory, and the University of Washington.
Adelman-McCarthy, J. K., et al. 2006, ApJS, 162, 38

Belokurov, V., et al. 2006, ApJ, 642, L137

Dehnen, W., \& Binney, J. 1998, MNRAS, 294, 429

Dinescu, D. I., Girard, T. M., van Altena, W. F., \& López, C. E. 2005, ApJ, 618, L25

Evans, N. W. 1993, MNRAS, 260, 191

Fellhauer, M., Kroupa, P., Baumgardt, H., Bien, R., Boily, C. M., Spurzem, R., \& Wassmer, N. 2000, NewA, 5, 305

Grebel, E. K. 2000, BAAS, 32, 698

Gunn, J. E., et al. 2006, AJ, 131, 2332

Helmi, A. 2004a, MNRAS, 351, 643 2004b, ApJ, 610, L97

Hogg, D. W., Finkbeiner, D. P., Schlegel, D. J., \& Gunn, J. E. 2001, AJ, 122, 2129

Ibata, R., Irwin, M., Lewis, G. F., \& Stolte, A. 2001a, ApJ, 547, 133L

Ibata, R., Lewis, G. F., Irwin, M., Totten, E., \& Quinn, T. 2001b, ApJ, 551, 294

Ibata, R. A., Gilmore, G., \& Irwin, M. J. 1994, Nature, 370, 194

Ibata, R. A., Wyse, R. F. G., Gilmore, G., Irwin, M. J., \& Suntzeff, N. B. 1997, AJ, 113, 634

Irwin, M., Ibata, R., Gilmore, G., Wyse, R., \& Suntzeff, N. 1996, in ASP Conf. Ser. 92, Formation of the Galactic Halo. ..Inside and Out, ed. G. W. Preston,

H. Morrison, \& A. Sarajedini (San Francisco: ASP), 84

Ivezić, Ž., et al. 2004, Astron. Nachr., 325, 583

\section{REFERENCES}

Johnston, K. V., Law, D. R., \& Majewski, S. R. 2005, ApJ, 619, 800

Law, D. R., Johnston, K. V., \& Majewski, S. R. 2005, ApJ, 619, 807

Majewski, S. R., Skrutskie, M. F., Weinberg, M. D., \& Ostheimer, J. C. 2003 , ApJ, 599, 1082

Majewski, S. R., et al. 2004, AJ, 128, 245

Navarro, J., Frenk, C. S., \& White, S. D. M. 1995, MNRAS, 275, 56

Neistein, E., van den Bosch, F., \& Dekel, A. 2006, MNRAS, in press (astro-ph/ 0605045)

Newberg, H. J., et al. 2002, ApJ, 569, 245 2003, ApJ, 596, L191

Olling, R. P., \& Merrifield, M. 2000, MNRAS, 311, 361

Pier, J. R., Munn, J. A., Hindsley, R.B, Hennessy, G. S., Kent, S. M., Lupton, R. H., \& Ivezic, Z. 2003, AJ, 125, 1559

Sevenster, M. 1999a, MNRAS, 310, 629

. 1999b, Ap\&SS, 265, 377

Skrutskie, M. F., et al. 2006, AJ, 131, 1163

Smith, J. A., et al. 2002, AJ, 123, 2121

Stoughton, C., et al. 2002, AJ, 123, 485

Totten, E. J., \& Irwin, M. J. 1998, MNRAS, 294, 1

van den Bosch, F. 2002, MNRAS, 331, 98

van der Marel, R. P. 1991, MNRAS, 248, 515 